\title{
Flagellar Synchronization Independent of Hydrodynamic Interactions
}

\author{
Benjamin M. Friedrich* and Frank Jülicher \\ Max Planck Institute for the Physics of Complex Systems, Nöthnitzer Straße 38, 01187 Dresden, Germany
}

(Received 7 June 2012; published 24 September 2012)

\begin{abstract}
Inspired by the coordinated beating of the flagellar pair of the green algae Chlamydomonas, we study theoretically a simple, mirror-symmetric swimmer, which propels itself at low Reynolds number by a revolving motion of a pair of spheres. We show that perfect synchronization between these two driven spheres can occur due to the motion of the swimmer and local hydrodynamic friction forces. Hydrodynamic interactions, though crucial for net propulsion, contribute little to synchronization for this free-moving swimmer.
\end{abstract}

DOI: 10.1103/PhysRevLett.109.138102

PACS numbers: 87.16.Qp, 05.45.Xt, 47.63.-b

Eukaryotic flagella are whiplike cell appendages that can bend actively, propel microorganisms at low Reynolds numbers, and pump fluids, e.g., mucus in our airways [1]. Their active bending waves are generated by a highly conserved cytoskeletal core with cylindrically arranged microtubules intercalated by molecular motors [2] that convert chemical energy into work and heat. Mechanical interactions are thought to underlie the coordinated beating of several flagella as observed in pairs of sperm cells [3] or in ciliary arrays, where hundreds of short flagella beat in synchrony as metachronal waves [4]. Recently, the biflagellate green alga Chlamydomonas has emerged as an experimental model system for flagellar synchronization [5-7]. A Chlamydomonas cell swims forward by the approximately planar and mirror-symmetric bending waves of its two flagella, thus resembling a breast swimmer [8]; see Fig. 1(a). The synchronous beating of the two flagella is important for swimming along a straight path [9]. Free swimming cells often exhibit synchronized flagellar beating [8,9], raising the question of the underlying synchronization mechanism. For flagella attached to a solid substrate, long-range hydrodynamic interactions can induce flagellar synchronization [10-16]. Synchronization of the flagella of a moving swimmer, however, shows different features: Here, we show that flagellar synchronization can occur as a result of local hydrodynamic friction forces, even in the absence of hydrodynamic interactions.

A model swimmer for biflagellar synchronization.Inspired by Chlamydomonas swimming, we propose a model swimmer of maximal simplicity that retains its basic symmetries. The swimmer consists of three spheres of equal radius $a$ and respective positions $\mathbf{r}_{j}=\left(x_{j}, y_{j}, 0\right)$ attached to a planar and mirror-symmetric scaffold; see Fig. 1(b). The swimmer is immersed in a viscous fluid of viscosity $\eta$ and the swimmer's scaffold is frictionless. The sphere located at $\mathbf{r}_{3}$ mimics a cell body and defines a material frame of the swimmer with orthonormal vectors $\mathbf{e}_{1}=\left(\cos \alpha_{3}, \sin \alpha_{3}, 0\right), \mathbf{e}_{3}=(0,0,1)$, and $\mathbf{e}_{2}=\mathbf{e}_{3} \times \mathbf{e}_{1}$, where the angular variable $\alpha_{3}$ characterizes rotations of the swimmer (around the $z$ axis) with respect to the $(x, y, z)$ laboratory frame; see Fig. 1(b).

The first and the second sphere move along circular orbits of radius $R, \mathbf{r}_{i}=\mathbf{s}_{i}+R\left(-\sin \varphi_{i} \mathbf{e}_{1}+\cos \varphi_{i} \mathbf{e}_{2}\right), i=1,2$, being connected by frictionless lever arms to joints located at the corners $\mathbf{s}_{i}=\mathbf{r}_{3}+l\left[(-1)^{i} \mathbf{e}_{1}+\mathbf{e}_{2}\right]$ of an isosceles triangle; see Fig. 1(b). Thus, $l$ sets the size of the swimmer. The orbits are parametrized by respective phase angles $\varphi_{i}$, $i=1,2$ such that $\left\lfloor\left(\varphi_{i}(t)-\varphi_{i}(0)\right) /(2 \pi)\right\rfloor$ denotes the number of full rotations of the $i$ th sphere since time $t=0$ with respect to the material frame of the swimmer. Similarly, $\left\lfloor\left(\alpha_{i}(t)-\alpha_{i}(0)\right) /(2 \pi)\right\rfloor$ with $\alpha_{i}=\alpha_{3}+\varphi_{i}$ denotes the number of rotations with respect to the laboratory frame. Below, the dynamics of the phase angles $\varphi_{i}$ is given in terms of active driving torques; the cases $\dot{\varphi}_{i}<0$ and $\dot{\varphi}_{i}>0$ correspond to either a clockwise or counterclockwise revolution of the driven spheres (viewed along $-\mathbf{e}_{3}$ ), respectively. The revolving motion of these driven spheres provides a simplified representation of the periodic bending waves of the two slender flagella of Chlamydomonas $[10,11]$, for which each point on a flagellum follows a

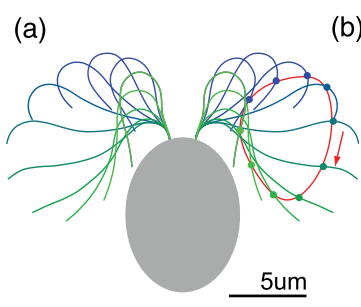

(b)

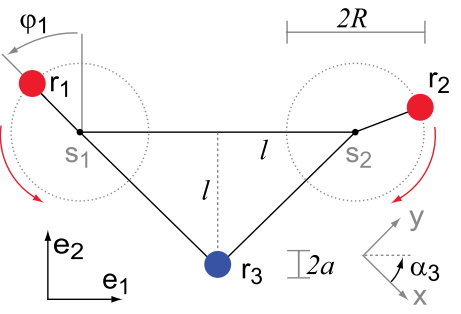

FIG. 1 (color online). (a) Simplified flagellar beat of Chlamydomonas showing flagellar shapes at equidistant times representing a full beat cycle $(T \approx 15 \mathrm{~ms})$, adapted from [34]. The flagellar bending waves are approximately planar and mirror-symmetric. Each point on a flagellum moves on a periodic orbit with respect to a material frame of the cell body. (b) The idealized model swimmer consists of three equal spheres connected by a frictionless scaffold. The first and second sphere (located at $\mathbf{r}_{1}$ and $\mathbf{r}_{2}$ ) can move along a circular trajectory as indicated, being driven by internally generated active torques. (The arrows correspond to the case $\omega_{0}>0$ ). 
periodic orbit in a material frame of the cell body; see Fig. 1(a).

We neglect inertial effects, which implies that fluid flow is governed by the Stokes equation of zero Reynolds number hydrodynamics $[1,17]$. We consider the hydrodynamic friction force $\mathbf{F}_{j}$ and torque $\mathbf{T}_{j}$ (defined with respect to $\mathbf{r}_{3}$ ) exerted by the $j$ th sphere on the viscous fluid during motion of the swimmer; $\mathbf{T}_{j}^{\prime}=\mathbf{T}_{j}-\mathbf{F}_{j} \times\left(\mathbf{r}_{j}-\mathbf{r}_{3}\right)$ denote torques with respect to $\mathbf{r}_{j}$. For free swimming, force and torque balance holds, $\mathbf{F}_{\text {ext }}=0$ and $\mathbf{T}_{\text {ext }}=0$ with $\mathbf{F}_{\text {ext }}=$ $\mathbf{F}_{1}+\mathbf{F}_{2}+\mathbf{F}_{3}, \mathbf{T}_{\text {ext }}=\mathbf{T}_{1}+\mathbf{T}_{2}+\mathbf{T}_{3}$. The linearity of the Stokes equation implies a linear relationship between the generalized velocity vector for planar motion of the three spheres, $\dot{\mathbf{q}}_{0}$ with $\mathbf{q}_{0}=\left(x_{1}, y_{1}, \alpha_{1}, \ldots, x_{3}, y_{3}, \alpha_{3}\right)^{T}$, and the nonzero hydrodynamic friction force and torque components [17],

$$
\left(F_{1 x}, F_{1 y}, T_{1 z}^{\prime}, \ldots, F_{3 x}, F_{3 y}, T_{3 z}^{\prime}\right)^{T}=\Gamma_{0} \dot{\mathbf{q}}_{0} .
$$

The symmetric $9 \times 9$ hydrodynamic friction matrix $\Gamma_{0}$ can be computed to arbitrary precision in $a /\left|\mathbf{r}_{j}-\mathbf{r}_{k}\right|$ [18]; in the limit of large separation between the spheres, the friction matrix would be diagonal, $\Gamma_{0, i j}=\gamma_{j} \delta_{i j}$, with $\gamma_{j}=\gamma_{\text {rot }}$ for $j=3,6,9$ and $\gamma_{j}=\gamma$ otherwise. Here, $\gamma=6 \pi \eta a$ and $\gamma_{\text {rot }}=8 \pi \eta a^{3}$ denote the translational and rotational friction coefficients of a single sphere of radius $a$, respectively. In general, the flow field induced by the motion of one sphere will exert forces on the other spheres, thus giving rise to nonzero, off-diagonal components of $\Gamma_{0}$, which characterize hydrodynamic interactions between the spheres. Below, we use the Rotne-PragerYamakawa approximation for $\Gamma_{0}=\Gamma_{0}\left(q_{0}\right)$, which generalizes the Oseen tensor and applies to both translational and rotational motion [18].

The swimmer is characterized by 5 degrees of freedom, represented by a vector of generalized coordinates, $\mathbf{q}=\left(x_{3}, y_{3}, \alpha_{3}, \varphi_{1}, \varphi_{2}\right)$, if its internal constraints are taken into account. In the following, we use the framework of Lagrangian mechanics of dissipative systems [19] to describe the dynamics of our swimmer. First, $\dot{\mathbf{q}}_{0}=L \dot{\mathbf{q}}$, with a $9 \times 5$ transformation matrix $L_{i j}=\partial q_{0 i} / \partial q_{j}$. The rate $\mathcal{R}_{h}=\dot{\mathbf{q}}_{0}{ }^{T} \Gamma_{0} \dot{\mathbf{q}}_{0}$ of hydrodynamic energy dissipation during swimming can be equivalently written as $\mathcal{R}_{h}=\dot{\mathbf{q}}^{T} \Gamma_{h} \dot{\mathbf{q}}$ with the $5 \times 5$ friction matrix $\Gamma_{h}=L^{T} \Gamma_{0} L$. The energy for active swimming is provided by a fuel reservoir, which we take for simplicity as infinite with internal energy $U=-m_{1} \varphi_{1}-m_{2} \varphi_{2}$. Here, $m_{1}$ and $m_{2}$ denote active driving torques that are assumed to be independent of the present phase $\varphi_{i}$ (but see [16]). The potential $U$ defines generalized potential forces $Q_{j}=-\partial U / \partial q_{j}, j=1, \ldots, 5$. Further, we introduce the Rayleigh dissipation function $\mathcal{R}=\mathcal{R}_{h}+\mathcal{R}_{\kappa}$ that sets the rate at which the energy reservoir is depleted, $-\dot{U}=\mathcal{R}$. Here, $\mathcal{R}_{\kappa}=\kappa\left(\dot{\varphi}_{1}^{2}+\dot{\varphi}_{2}^{2}\right)$ denotes a rate of internal dissipation associated with the actuation of the two driven spheres. For notational convenience, $\quad \mathcal{R}_{\kappa}=\dot{\mathbf{q}}^{T} \Gamma_{\kappa} \dot{\mathbf{q}}$ with $\quad \Gamma_{\kappa, i j}=0 \quad$ except
$\Gamma_{\kappa, 44}=\Gamma_{\kappa, 55}=\kappa$. The generalized friction forces $P_{i}=(1 / 2) \partial \mathcal{R} / \partial \dot{q}_{i}$ are linear in $\dot{\mathbf{q}}, P_{i}=\Gamma_{i j} \dot{q}_{j}$ with $\Gamma=\Gamma_{h}+\Gamma_{\kappa}$. Neglecting inertial forces and assuming that no external forces act on the swimmer, we find a balance of generalized potential forces and generalized friction forces $Q_{j}=P_{j}, j=1, \ldots, 5$. For example, the equation for $j=4$, corresponding to $q_{4}=\varphi_{1}$, represents a torque balance between a hydrodynamic friction torque, $(1 / 2) \partial \mathcal{R}_{h} / \partial \dot{\varphi}_{1}=\mathbf{F}_{1} \cdot\left(\partial \mathbf{r}_{1} / \partial \varphi_{1}\right)+T_{1 z}^{\prime}$, and a net motor torque $m_{1}-\kappa \dot{\varphi}_{1}$ provided by active driving. This motor torque obeys a linear torque-velocity relation with stall torque $m_{1}$, similar to the net driving force used in [10]. An analogous statement holds for the second sphere. We finally obtain an equation of motion of the swimmer [20],

$$
\dot{\mathbf{q}}=\Gamma^{-1}\left(0,0,0, m_{1}, m_{2}\right)^{T} .
$$

We first discuss the case of exactly opposite driving torques $m_{1}=-m_{2}$, which results in a counterrotation of sphere 1 and sphere 2 [Fig. 1(b)], similar to the mirror-symmetric beat patterns of the two flagella of Chlamydomonas [Fig. 1(a)]. The angular frequency $\omega_{0}=$ $m_{1} / \kappa$ sets an (inverse) time scale of motion. If $\omega_{0}>0$, the revolution of the first sphere is counterclockwise and clockwise for the second. The two cases $\omega_{0}>0$ and $\omega_{0}<0$ are mapped onto each other by time reversal.

Net propulsion due to hydrodynamic interactions.-For $m_{1}=-m_{2}$, there exists an orbit with perfect in-phase dynamics characterized by $\delta=0$, where $\delta=\varphi_{1}+\varphi_{2}$. Below, we show that this orbit is stable for $\omega_{0}<0$, but unstable for $\omega_{0}>0$. If initially $\alpha_{3}(t=0)=0$, the swimmer will move parallel to the $y$ axis in an oscillatory manner: In the limit of small spheres and small circular orbits, $a \ll l, \quad R \ll l$, we find to leading order $\dot{y}=(2 / 3) R \omega_{0} \sin \varphi_{1}+\mathcal{O}\left(\varepsilon^{2}\right)$ with $\dot{\varphi}_{1}=\omega_{0}+\mathcal{O}\left(\varepsilon^{3}\right)$. Here, we introduced the small expansion parameter $\varepsilon=a / l$ and assume $R / a$ to be of order unity. In this limit, internal dissipation dominates over hydrodynamic dissipation, $\kappa \gg \gamma R^{2}+\gamma_{\text {rot }}$. Net propulsion is a higher-order effect $[21,22]$ and the time-averaged velocity reads

$$
\langle\dot{y}\rangle=-a \omega_{0} \Theta(a / l)^{2}+\mathcal{O}\left(\varepsilon^{4}\right),
$$

with $\Theta=\left[(2 \sqrt{2}-1)(R / a)^{2}-4(1+\sqrt{2})\right] / 24$. Note that the swimmer can move either forward or backward depending on the value of $R / a$ and the sign of $\omega_{0}$. For asynchronous beating with $\delta=\varphi_{1}+\varphi_{2} \neq 0, \alpha_{3}$ oscillates and the swimmer wiggles along a curved path.

If hydrodynamic interactions were absent, i.e. $\Gamma_{0, i j}=$ $\gamma_{j} \delta_{i j}$, the center of reaction $\mathbf{r}_{c}=\sum_{j} \mathbf{r}_{j} / 3$ of the swimmer could not move since $3 \gamma \dot{\mathbf{r}}_{c}=\mathbf{F}_{\text {ext }}=0$, and net propulsion would be zero. This is a well-known feature of hydrodynamics at zero Reynolds number. Partial screening of hydrodynamic interactions can occur, e.g., for swimming close to a planar substrate.

A system of coupled phase oscillators.-The phase velocities $\dot{\varphi}_{1}$ and $\dot{\varphi}_{2}$ cannot depend on the momentary 
values of $x_{3}, y_{3}, \alpha_{3}$, as the phase variables are invariant under a change of laboratory frame, but the position and orientation variables are not. Indeed, one can eliminate the latter from Eq. (2) and obtain a dynamical system for $\varphi_{1}$ and $\varphi_{2}$ in the form of two coupled phase oscillators,

$$
\kappa \dot{\varphi}_{i}=m_{i}+\varepsilon^{3} h_{i 1}\left(\varphi_{1}, \varphi_{2}\right) m_{1}+\varepsilon^{3} h_{i 2}\left(\varphi_{1}, \varphi_{2}\right) m_{2}, \quad i=1,2 .
$$

Here the coupling functions $h_{i j}$ depend only on $\left[\varphi_{i}\right]=$ $\varphi_{i} \bmod 2 \pi$ and can be computed from the friction matrix $\Gamma$. Importantly, the $h_{i j}$ implicitly account for the motion of the swimmer and imposing constraints on the motion would change these functions.

Synchronization of counterrotating spheres.-For opposite driving torques, $m_{1}=-m_{2}=\omega_{0} \kappa$, we find two limit cycles of the phase dynamics characterized by $\delta=0$ and $\delta=\pi+\mathcal{O}\left(\varepsilon^{3}\right)$, respectively, where $\delta=\varphi_{1}+\varphi_{2}$. The first limit cycle corresponds to perfect in-phase dynamics as considered in the paragraph on net propulsion and is a global attractor for $\omega_{0}<0$, but unstable for $\omega_{0}>0$; see Fig. 3(b). Limit cycles of the $\left(\varphi_{1}, \varphi_{2}\right)$ phase dynamics correspond to fixed points of a Poincaré return map $\delta_{n} \rightarrow$ $\delta_{n+1}=\delta_{n}+\Lambda\left(\delta_{n}\right)$ that tracks $\delta_{n}=\delta\left(t_{n}\right)$ at discrete times $t_{n}$ that mark the completion of $n$ full revolutions of the first sphere, $\varphi_{1}\left(t_{n}\right)=2 \pi n \operatorname{sgn}\left(\omega_{0}\right)$. The solid curve in Fig. 2(a) shows a numerical solution for $\Lambda(\delta)$ as a function of initial phase sum $\delta=\delta_{0}$. By symmetry, $\Lambda(0)=0$, which corresponds to the limit cycle of in-phase synchronization with $\delta=0$. This limit cycle will be stable if
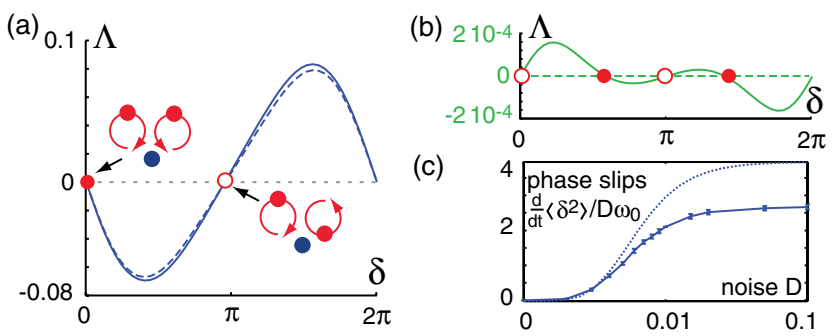

FIG. 2 (color online). (a) Phase synchronization behavior of our model swimmer (here for $m_{1}=-m_{2}<0$ ) can be read off from a Poincaré return map $\Lambda=\delta(T)-\delta(0)$ that tracks the change of the phase sum $\delta=\varphi_{1}+\varphi_{2}$ after a full revolution of the first sphere as function of initial $\delta=\delta(t=0)$. Fixed points $\Lambda=0$ correspond to limit cycles of the $\left(\varphi_{1}, \varphi_{2}\right)$-phase dynamics; see Fig. 3(b1). For the dashed and solid curve, hydrodynamic interactions were neglected or accounted for, respectively. (b) Constraining translation and rotation of the swimmer by clamping the third sphere changes its synchronization behavior completely. (c) In the presence of fluctuations, the phase dynamics of the free swimmer exhibits stochastic phase slips that occur at a frequency $(2 \pi)^{-2} d\left\langle\delta^{2}\right\rangle / d t$; shown is $d\left\langle\delta^{2}\right\rangle / d t$ normalized by $D \omega_{0}$ (solid curve), as well as the analytical result [25] $d\left\langle\delta^{2}\right\rangle / d t \approx 4 D \omega_{0} I_{0}^{-2}[\lambda /(4 \pi D)]$ for the approximate dynamics given by Eq. (6), (dotted curve). Parameters: $a / l=0.1$, $R / a=5, \kappa=\eta l^{3}$. $\lambda=-d \Lambda / d \delta_{\mid \delta=0}$ is positive. In the limit of small spheres and small circular orbits, $\Lambda(\delta)=-\lambda \sin \delta+\mathcal{O}\left(\varepsilon^{6}\right)$ with

$$
\lambda=-\operatorname{sgn}\left(\omega_{0}\right) 3 \pi \gamma R^{4} /\left(16 \kappa l^{2}\right)+\mathcal{O}\left(\varepsilon^{6}\right) .
$$

Thus, in-phase synchronization with $\delta=0$ is stable for $\omega_{0}<0$ and perturbations decay as $\lim _{n \rightarrow \infty} \delta_{n+1} / \delta_{n}=$ $1-\lambda$.

Remarkably, if hydrodynamic interactions are neglected, $\Lambda(\delta)$ does not change significantly; see Fig. 2(a) (dashed curve). In fact, hydrodynamic interactions contribute to $\Lambda(\delta)$ only to higher order as $\mathcal{O}\left(\varepsilon^{6}\right)$. This implies that hydrodynamic interactions have only a marginal effect for the phase synchronization of our model swimmer. Rather, local hydrodynamic friction forces that arise from the motion of the swimmer dominate synchronization: If one driven sphere is ahead of the other, this asynchronous beating results in a rotation of the whole swimmer, accompanied by hydrodynamic friction forces acting on the spheres.

In the presence of constraining forces that prevent the swimmer from translating and rotating, the coupling functions $h_{i j}$ in Eq. (4) change, resulting in weak synchronization toward novel limit cycles; see Fig. 2(b). In this case, synchronization is due to hydrodynamic interactions only.

For a microscopic oscillator such as a beating flagellum powered by molecular motors, noise is prevalent and may counteract synchronization. As a simple model for motor fluctuations, we now consider fluctuating driving torques, $m_{1}=k \omega_{0}+\xi_{1}$ and $m_{2}=-k \omega_{0}(1+v)+\xi_{2},\left\langle\xi_{i}\right\rangle=0$, together with a detuning of driving torques, $v$. We assume a noise correlation time short compared to $T=2 \pi /\left|\omega_{0}\right|$, and model $\xi_{i}$ as Gaussian white noise with $\left\langle\xi_{i}(t) \xi_{j}\left(t^{\prime}\right)\right\rangle=2 D \omega_{0} \kappa^{2} \delta_{i j} \delta\left(t-t^{\prime}\right)$, where $D$ denotes a dimensionless noise strength. Equation (4) thus becomes a stochastic equation with multiplicative noise (for which Stratonovich interpretation is to be used). For weak noise with $D \ll 1$, the behavior of $\delta=\varphi_{1}+\varphi_{2}$, averaged over cycles of the fast variable $\varphi_{1} \approx \omega_{0} t$, is to a good approximation [23] given by the prototypical Adler equation [24-26],

$$
d \delta / d t \approx v \omega_{0}-(\lambda / T) \sin \delta+\xi,
$$

where $\xi$ denotes Gaussian white noise with $\left\langle\xi(t) \xi\left(t^{\prime}\right)\right\rangle=$ $4 D \omega_{0} \delta\left(t-t^{\prime}\right)$. Equation (6) describes a Brownian particle with position $\delta$ that diffuses in a tilted washboard potential. For $v, D \ll|\lambda|, \delta$ fluctuates within one potential well (corresponding to transient synchronization), with occasional phase slips from one well to the next. For $v=0$, the frequency $(2 \pi)^{-2} d\left\langle\delta^{2}\right\rangle / d t$ of these phase slips scales with noise strength $D$ for $D \gg|\lambda|$, but is suppressed for $D \ll|\lambda|$ [25]; see Fig. 2(c). In the case of torque detuning, $v \neq 0$, phase locking can occur as discussed next.

In the general case of arbitrary driving torques $m_{1}$ and $m_{2}$ (and no noise), phase locking between $\varphi_{1}$ and $\varphi_{2}$ can occur, i.e. $n_{2} \varphi_{1}-n_{1} \varphi_{2}$ remains bounded for some choice of integers $n_{1}, n_{2}$. Figure 3(a) shows parameter regions 


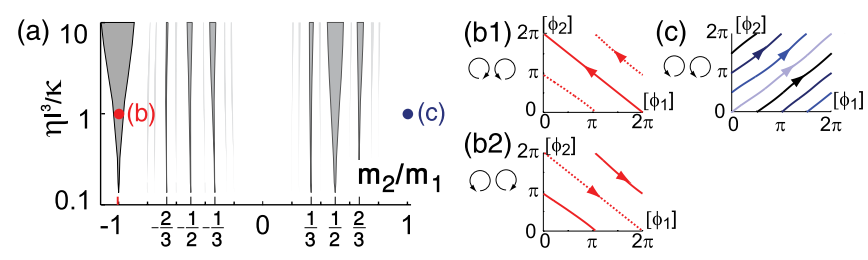

FIG. 3 (color online). (a) Depending on the ratio of the active driving torques $m_{1}$ and $m_{2}$, phase locking of the phase variables $\varphi_{1}$ and $\varphi_{2}$ describing our model swimmer can occur giving rise to a distinct pattern of Arnold tongues. (b) As specific example, a limit cycle with $\delta=\varphi_{1}+\varphi_{2}=0$ is globally attractive for opposite torques with $m_{1}=-m_{2}<0$ (b1), but repulsive for $m_{1}=-m_{2}>0$ (b2). (c) For equal torques $m_{1}=m_{2}$, the $\left(\varphi_{1}, \varphi_{2}\right)$ phase space is foliated by neutrally stable orbits. Parameters: $a / l=0.1, R / a=5, \kappa=\eta l^{3}$.

(called Arnold tongues [26]) for which phase locking occurs.

The case of corotating spheres with $m_{1}=m_{2}$ is special in the sense that the dynamics becomes reversible: The mirror operation $\left(\varphi_{1}, \varphi_{2}\right) \rightarrow\left(-\varphi_{2},-\varphi_{1}\right)$ maps each orbit onto itself, but reverses the time arrow. The $\left(\varphi_{1}, \varphi_{2}\right)$ phase space is foliated by neutrally stable orbits; see Fig. 3(c). Hence, for identical driving torques, no specific phase difference is selected. In the presence of a small mismatch $v$ in driving torque, $m_{2}=m_{1}(1+v)$ with $|v| \ll 1$, we find $\Lambda(\delta)=2 \pi v+\mathcal{O}\left(v \varepsilon^{3}\right) \bmod 2 \pi$, which rules out the possibility of $2 \pi$-periodic orbits for $v \neq 0$ and therefore synchronization cannot occur in this case.

Generally, symmetries dictate synchronization behavior [27]. For two interacting oscillators, time reversal changes an attractor of their phase dynamics such as a synchronized state into a repeller. If the time-reversed system is equivalent to a mirror image of itself, stable synchronization can therefore not occur [27]. This is exemplified by our swimmer with corotating spheres $\left(m_{1}=m_{2}\right)$; see Fig. 3(c). Our swimmer with counterrotating spheres $\left(m_{1}=-m_{2}\right)$ is, after a time reversal, not equivalent to its mirror image. Correspondingly, there exist limit cycles with synchronized dynamics in this case, and their stability reverses under time reversal; see Fig. 3(b). Note that in the absence of the third sphere, time reversal would be equivalent to a reflection at the plane that contains both rotation axes and synchronization would be lost for our model swimmer with its rigid scaffold. Previous research demonstrated that elasticity of the rotating objects introduces additional degrees of freedom that can break symmetries and thus stabilize synchronization $[11,12]$. However, reversibility may also be broken without evoking elasticity as in the case of our three-sphere swimmer.

A realistic flagellar beat.-The conceptual framework of our model swimmer can be extended in a straightforward manner to any mirror-symmetric microswimmer whose swimming stroke is characterized by two phase angles $\varphi_{1}$ and $\varphi_{2}$. As an example, consider the idealized flagellar beat in Fig. 1(a): During a beat cycle, the centerline $\mathbf{r}_{i}$ of
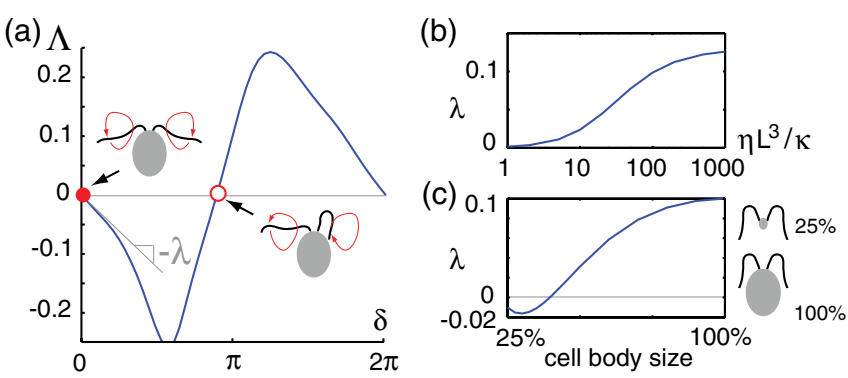

FIG. 4 (color online). Flagellar synchronization of Chlamydomonas computed for the beat pattern from Fig. 1(a) using Eq. (7) and resistive force theory. (a) The Poincaré return map $\Lambda(\delta)$ [defined analogous to that of Fig. 2(a)] shows that in-phase flagellar beating with $\delta=0$ is stable with respect to perturbations. (b),(c) The synchronization parameter $\lambda=$ $-d \Lambda / d \delta_{\mid \delta=0}$ depends on the ratio of hydrodynamic dissipation and internal dissipation (b) as well as on the size of the cell body (c). Parameters (unless indicated otherwise): flagellar length, $L=12 \mu \mathrm{m}$; semiaxes of spheroidal cell body $3.7 \mu \mathrm{m}$, $5 \mu \mathrm{m}$ [34]; resistive force coefficients, $\zeta_{\|}=2 \pi \eta /[\ln (2 L / r)-$ $3 / 2], \zeta_{\perp}=4 \pi \eta /[\ln (2 L / r)-3 / 2]$ [29] with flagellar radius $r=0.1 \mu \mathrm{m}[2], \eta L^{3} / \kappa=100$.

each of the two flagella can be expressed as a function of arclength $s, 0 \leq s \leq L$, and phase angles $\varphi_{i}, i=1$, 2, with $\dot{\varphi}_{1}>0$ and $\dot{\varphi}_{2}<0$ that characterize the phase of the beat cycle: With respect to a material frame $\left(\mathbf{r}_{3} ; \mathbf{e}_{1}, \mathbf{e}_{2}, \mathbf{e}_{3}\right)$ of the cell body, $\mathbf{r}_{i}\left(s, \varphi_{i}\right)=\mathbf{r}_{3}+c_{i 1}\left(s, \varphi_{i}\right) \mathbf{e}_{1}+c_{i 2}\left(s, \varphi_{i}\right) \mathbf{e}_{2}$, $i=1,2$. By mirror symmetry of the two flagella, $c_{1 j}(s, \varphi)=(-1)^{j} c_{2 j}(s,-\varphi)$. We make the simplifying assumption that hydrodynamic forces do not alter the sequence of flagellar shapes (i.e. the shape functions $c_{i j}$ ), but only affect the phase speeds $\dot{\varphi}_{i}$.

The force balance equations for the dynamics of the three-sphere swimmer generalize to the case of a realistic flagellar beat; the torque balance corresponding to $\varphi_{i}$ now reads [28]

$\int_{0}^{L} d s \mathbf{f}_{i}(s) \cdot \partial \mathbf{r}_{i}(s) / \partial \varphi_{i}=m_{i}-\kappa \dot{\varphi}_{i}, \quad i=1,2$.

To compute the density $\mathbf{f}_{i}(s)$ of hydrodynamic friction forces along the flagellar length as a function of $\varphi_{i}$ and $\dot{\varphi}_{i}$, we employ simple resistive force theory [29-32], while the cell body is approximated by a drag center equivalent to a spheroid.

Resistive force theory accounts for short-range hydrodynamic effects along a single flagellum by assuming effective anisotropic friction coefficients. This is crucial for net propulsion. However, hydrodynamic interactions between the two flagella, or with the cell body are not accounted for. Despite these approximations, we compute realistic, saltatory forward swimming for in-phase flagellar beating with a net swimming speed of $0.66 \mu \mathrm{m} /$ cycle. As a main result, perfect flagellar synchronization with $\delta=0$ is stable; see Fig. 4(a). Of note, the synchronization parameter $\lambda$ has different sign in the case of the realistic 
flagellar beat and that of the simple three-sphere swimmer with $m_{1}=-m_{2}>0$; this can be related to geometric factors such as cell body size; see Fig. 4(c).

Discussion.-Using a minimal model, we have demonstrated that synchronization can occur between the two flagella of a free-moving swimmer due to the motion of the swimmer itself, independent of hydrodynamic interactions between the flagella. Importantly, the synchronization behavior changes fundamentally if the swimmer is restrained from translating and rotating. Local hydrodynamic friction forces couple the flagellar oscillators via movements of the swimmer, even in the absence of hydrodynamic interactions. Coupling of oscillators by swimmer movements can be strongly influenced by externally imposed constraints, which typically exist in experiments. For our model swimmer, synchronization of the flagellar phases attenuates if the swimmer is restrained from translating and rotating. In Chlamydomonas, perfect in-phase synchronization of its two flagella was reported also for cells held in a micropipette [5-7], and even for isolated flagellar pairs detached from the cell body [33]. Hydrodynamic interactions between the two flagella and flexibility of the flagellar beat, as proposed by others $[10,11]$, may contribute to synchronization. We anticipate that synchronization depends sensitively on elastic properties of the flagellar base.

We thank J. Baumgart, E. Fischer-Friedrich, V. Geyer, J. Howard, R. Ketzmerick, L. Morelli, and A. Vilfan for stimulating discussions.

Note added in proof.-After submission of this Letter, we learned from R. Bennett and R. Golestanian that they have independently developed a similar three-sphere model for Chlamydomonas [35].

*ben@pks.mpg.de

[1] E. Lauga and T. R. Powers, Rep. Prog. Phys. 72, 096601 (2009).

[2] B. Alberts, D. Bray, J. Lewis, M. Raff, K. Roberts, and J. D. Watson, Molecular Biology of the Cell (Garland Science, New York, 2002), 4th ed.

[3] D. M. Woolley, R. F. Crockett, W. D. I. Groom, and S. G. Revell, J. Exp. Biol. 212, 2215 (2009).

[4] M. J. Sanderson and M. A. Sleigh, J. Cell Sci. 47, 331 (1981).

[5] U. Rüffer and W. Nultsch, Cell Motil. Cytoskeleton 41, 297 (1998).
[6] R. E. Goldstein, M. Polin, and I. Tuval, Phys. Rev. Lett. 103, 168103 (2009).

[7] R. E. Goldstein, M. Polin, and I. Tuval, Phys. Rev. Lett. 107, 1 (2011).

[8] U. Rüffer and W. Nultsch, Cell Motil. 5, 251 (1985).

[9] M. Polin, I. Tuval, K. Drescher, J. P. Gollub, and R. E. Goldstein, Science 325, 487 (2009).

[10] A. Vilfan and F. Jülicher, Phys. Rev. Lett. 96, 058102 (2006).

[11] T. Niedermayer, B. Eckhardt, and P. Lenz, Chaos 18, 037128 (2008).

[12] M. Reichert and H. Stark, Eur. Phys. J. E 17, 493 (2005).

[13] S. Gueron and K. Levit-Gurevich, Proc. Natl. Acad. Sci. U.S.A. 96, 12240 (1999).

[14] M. Kim and T. R. Powers, Phys. Rev. E 69, 061910 (2004).

[15] J. Kotar, M. Leoni, B. Bassetti, M. C. Lagomarsino, and P. Cicuta, Proc. Natl. Acad. Sci. U.S.A. 107, 7669 (2010).

[16] N. Uchida and R. Golestanian, Phys. Rev. Lett. 106, 058104 (2011).

[17] J. Happel and H. Brenner, Low Reynolds Number Hydrodynamics (Kluwer, Boston, 1965).

[18] J. Dhont, An Introduction to Dynamics of Colloids (Elsevier, New York, 1996).

[19] H. Goldstein, C. Poole, and J. Safko, Classical Mechanics (Addison-Wesley, Reading, MA, 2002), 3rd ed.

[20] $\Gamma$ is always positive definite, while $\Gamma_{h}$ can become singular for special configurations $\mathbf{q}$

[21] A. Shapere and F. Wilczek, Phys. Rev. Lett. 58, 2051 (1987).

[22] A. Najafi and R. Golestanian, Phys. Rev. E 69, 062901 (2004).

[23] Specifically, if $\bar{\delta}$ is defined as the solution to Eq. (6) with $\bar{\delta}(0)=\delta(0)$, then after one cycle, $\bar{\delta}$ will have the same statistics as $\delta$ to leading order, $\left\langle\bar{\delta}^{n}(T)\right\rangle=\left\langle\delta^{n}(T)\right\rangle+$ $\mathcal{O}\left(\varepsilon^{3} v, \varepsilon^{3} D, \varepsilon^{6}\right)$.

[24] R. Adler, Proc. IRE 34, 351 (1946).

[25] R. L. Stratonovich, Topics in the Theory of Random Noise (Gordon and Breach, New York, 1963).

[26] A. Pikovsky, M. Rosenblum, and J. Kurths, Synchronization (Cambridge University Press, Cambridge, England, 2001).

[27] G. Elfring and E. Lauga, Phys. Rev. Lett. 103, 1 (2009).

[28] We neglect local torques corresponding to rotations of infinitesimal flagellar segments around their center.

[29] J. Gray and G. T. Hancock, J. Exp. Biol. 32, 802 (1955).

[30] B. M. Friedrich, I. H. Riedel-Kruse, J. Howard, and F. Jülicher, J. Exp. Biol. 213, 1226 (2010).

[31] P. V. Bayly, B. L. Lewis, E. C. Ranz, R. J. Okamoto, R. B. Pless, and S. K. Dutcher, Biophys. J. 100, 2716 (2011).

[32] M. S. Jones, L. Le Baron, and T. J. Pedley, J. Fluid Mech. 281, 137 (1994).

[33] J. S. Hyams and G. G. Borisy, Science 189, 891 (1975).

[34] C. J. Brokaw and D. J. Luck, Cell Motil. 3, 131 (1983).

[35] R. Bennett and R. Golestanian (private communication). 